\title{
Clinical risk factors for decompensated heart failure after atrial fibrillation ablation
}

\author{
Yasuhiro Matsuda ${ }^{1}$, Masaharu Masuda ${ }^{1}$, Takashige Sakio ${ }^{1}$, Mitsutoshi Asai $^{1}$, Osamu Iida ${ }^{1}$, \\ Shin Okamoto ${ }^{1}$, Takayuki Ishihara ${ }^{1}$, Kiyonori Nanto ${ }^{1}$, Takashi Kanda ${ }^{1}$, Takuya \\ Tsujimura $^{1}$, Yosuke Hata ${ }^{1}$, Hiroyuki Uematsu ${ }^{1}$, and Toshiaki Mano ${ }^{1}$ \\ ${ }^{1}$ Kansai Rosai Hospital
}

June 21, 2021

\begin{abstract}
Introduction: Although decompensated heart failure (DHF) can complicate catheter ablation of atrial fibrillation (AF), its incidence and risk factors have not been defined. We sought to investigate the incidence and risk factors for DHF in these patients. Methods: In total, 1004 consecutive patients who underwent initial ablation for AF (age, $68 \pm 10$ years old; females, 346 [34\%]; and persistent AF, 513 [51\%]) were enrolled. $\Delta$ heart rate, which was defined as heart rate after ablation minus heart rate before ablation, were calculated. DHF was defined as heart failure requiring medical therapy during post-procedure hospitalization, or re-hospitalization due to heart failure $<90$ days after the procedure. DHF was classified into early periprocedual DHF, which occurring within 2 days after the procedure, and late peri-procedual DHF, which occurring [?] 3 days after the procedure. Results: The incidence of DHF was 32/1004 (3\%) patients. Patients with DHF had a higher prevalence of a past history of symptomatic heart failure $(17 / 32[53 \%]$ versus $154 / 972[16 \%], \mathrm{P}<0.01)$ and lower $\Delta$ heart rate after the procedure than those without $(-16 \pm 28$ versus $2 \pm 21$ beats $/$ min, $\mathrm{P}<0.01)$. On multivariate analysis, lower $\Delta$ heart rate was a significant independent predictor of early peri-procedual DHF, while early recurrence of AF was a significant independent predictor of late peri-procedual DHF. Conclusion: In patients with AF, lower $\Delta$ heart rate was an independent predictor of early peri-procedual DHF, and early recurrence of AF was an independent predictor of late peri-procedual DHF.
\end{abstract}

\section{Clinical risk factors for decompensated heart failure after atrial fibrillation ablation}

Yasuhiro Matsuda, MD, Masaharu Masuda, MD, PhD *, Takashige Sakio, MD, Mitsutoshi Asai, MD, PhD, Osamu Iida, MD, Shin Okamoto, MD, Takayuki Ishihara, MD, Kiyonori Nanto, MD, Takashi Kanda, MD, Takuya Tsujimura, MD, Yosuke Hata, MD, Hiroyuki Uematsu, MD, and Toshiaki Mano, MD, PhD

\section{All authors:}

Kansai Rosai Hospital Cardiovascular Center, 3-1-69 Inabaso, Amagasaki, Hyogo 660-8511, Japan

*Corresponding author:

Masaharu Masuda

Kansai Rosai Hospital Cardiovascular Center

3-1-69 Inabaso, Amagasaki-shi, Hyogo, 660-8511, Japan

Tel: +81-6-6416-1221

Fax: +81-6-6419-1870 
E-mail: masuda-masaharu@kansaih.johas.go.jp

Abstract (244 words)

Introduction: Although decompensated heart failure (DHF) can complicate catheter ablation of atrial fibrillation $(\mathrm{AF})$, its incidence and risk factors have not been defined. We sought to investigate the incidence and risk factors for DHF in these patients.

Methods: In total, 1004 consecutive patients who underwent initial ablation for AF (age, $68 \pm 10$ years old; females, 346 [34\%]; and persistent AF, 513 [51\%]) were enrolled. $\Delta$ heart rate, which was defined as heart rate after ablation minus heart rate before ablation, were calculated. DHF was defined as heart failure requiring medical therapy during post-procedure hospitalization, or re-hospitalization due to heart failure $<$ 90 days after the procedure. DHF was classified into early peri-procedual DHF, which occurring within 2 days after the procedure, and late peri-procedual DHF, which occurring [?] 3 days after the procedure.

Results: The incidence of DHF was 32/1004 (3\%) patients. Patients with DHF had a higher prevalence of a past history of symptomatic heart failure $(17 / 32[53 \%]$ versus $154 / 972[16 \%], P<0.01)$ and lower $\Delta$ heart rate after the procedure than those without $(-16 \pm 28$ versus $2 \pm 21$ beats $/ \mathrm{min}, P<0.01)$. On multivariate analysis, lower $\Delta$ heart rate was a significant independent predictor of early peri-procedual DHF, while early recurrence of AF was a significant independent predictor of late peri-procedual DHF.

Conclusion: In patients with $\mathrm{AF}$, lower $\Delta$ heart rate was an independent predictor of early peri-procedual $\mathrm{DHF}$, and early recurrence of AF was an independent predictor of late peri-procedual DHF.

\section{Key words}

Atrial fibrillation; Catheter ablation; Decompensated heart failure; Complications; Heart rate

\section{Introduction}

Catheter ablation is one of several well-established therapies for atrial fibrillation (AF), ${ }^{1}$ but is a complex interventional procedure which is associated with a significant risk of complications. ${ }^{1}$ A previous study showed an overall incidence of complications of $6.3 \%,{ }^{2}$ and decompensated heart failure (DHF) can occur after catheter ablation. ${ }^{3}$

There is a wide variety of risk factors for DHF, such as overhydration, tachycardia, bradycardia, and blood pressure elevation. ${ }^{4}$ Irrigation fluid, sedation, and invasion may also cause DHF after catheter ablation. ${ }^{4}$ In addition, cardiac output decreases in more than one-third of patients with cardioversion of AF, recovering by degrees over four weeks. ${ }^{5}$ These factors are thought to induce DHF after the procedure, however, it is not easy to identify whether DHF occurs after catheter ablation in the clinical setting.

Here, we investigated the incidence of and risk factors for DHF in patients undergoing AF ablation.

\section{Methods}

Patients

The data of a total of 1004 consecutive patients undergoing initial ablation for AF at our hospital from December 2014 to December 2018 were retrospectively analyzed.

Exclusion criteria were age $<20$ years and incomplete standard electrophysiological studies following pulmonary vein $(\mathrm{PV})$ isolation.

This study complied with the Declaration of Helsinki. Written informed consent for catheter ablation and the use of data in this study was obtained from all patients, and the protocol was approved by our institutional review board (Reference number 2001030).

Catheter ablation procedure 
Electrophysiological studies and catheter ablation were performed by experienced operators under intravenous sedation with propofol or dexmedetomidine. Electroanatomical mapping system (Carto 3, [Biosense Webster, Inc., Diamond Bar CA, USA]; Ensite NavX, [Abbott, Abbott Park IL, USA]; or Rhythmia, [Boston Scientific, Boston MA, USA]) were used. Radiofrequency catheter ablation was performed in all cases from October 2014 to March 2016. From March 2016 to December 2018 cryoballoon ablation was performed for paroxysmal $\mathrm{AF}$ and for persistent $\mathrm{AF}$ in patients considered frail and at high risk of procedure-related complications. Patients with common PVs or a large PV diameter underwent radiofrequency catheter ablation. Five patients with paroxysmal AF underwent laser balloon ablation from July 2018 to September 2018.

In general, Ringer's acetate was used as a peri-procedural infusion. Infusion volume before the procedure was $0.45 \mathrm{~L}$, infusion volume during the procedure was $0.1 \mathrm{~L} / \mathrm{h}$ and infusion volume after the procedure was 1 L. Peri-procedural infusion was reduced or avoided if hydration was contraindicated, such as in hemodialysis patients, or in other situations as per the operator's judgment.

In radiofrequency catheter ablation, circumferential ablation around both ipsilateral PVs was performed using an open-irrigated linear ablation catheter via a Swartz Braided SL0 Transseptal Guiding Introducer Sheath (Abbott) or AGILIS NXT Steerable Introducer (Abbott). PV isolation was considered complete when the 20-pole circular catheter no longer recorded any PV potentials.

In cryoballoon ablation, an Arctic Front Advance cryoballoon catheter with a 28-mm balloon size (Medtronic, Inc., Minneapolis MN, USA) was passed into each PV under guidance by fluoroscopy and/or a electroanatomical mapping system. After confirming PV occlusion by pulmonary venography, cryoablation commenced and was usually continued for 180 seconds. If left atrium-PV conduction persisted after cryoballoon ablation, an additional touch-up ablation was performed using an open-irrigated linear ablation catheter with a 3.5-mm tip.

Additional ablation was also performed for any AF triggers originating from non-PV foci induced by isoproterenol infusion, and for spontaneous atrial flutter or atrial tachycardia induced by atrial burst stimuli. Empirical ablation, such as left atrial linear ablation, complex fractionated atrial electrogram ablation, or low-voltage-area (LVA) ablation was also performed according to the operator's judgment.

Following PV isolation, voltage mapping was performed using a multi-electrode mapping catheter or bipolar 3.5-mm tip catheter during sinus rhythm or with pacing from the right atrium. The presence of LVAs was defined as areas with voltage $<0.5 \mathrm{mV}$ covering [?] $5 \mathrm{~cm}^{2}$ across the total surface area of the left atrium.

Thermocool SmartTouch (Biosense Webster), Thermocool SmartTouch SF (Biosense Webster), NAVISTAR Thermocool (Biosense Webster), Thermocool SF (Biosense Webster), CELSIUS (Biosense Webster), TactiCath SE (Abbott), or FlexAbility (Abbott) were used for open-irrigated linear ablation catheters. RF energy was applied for $30 \mathrm{~s}$ at each site using a maximum temperature of $42^{\circ} \mathrm{C}$ and maximum power of 35 W. An irrigation flow rate of $17 \mathrm{~mL} / \mathrm{min}$ was used with Thermocool SmartTouch, NAVISTAR Thermocool, or TactiCath SE catheters; $8 \mathrm{~mL} / \mathrm{min}$ was used with the other catheters.

\section{Followup}

DHF was defined as heart failure requiring medical therapy during post-procedure hospitalization, or that required re-hospitalization $<90$ days after the procedure. DHF was classified into early peri-procedural DHF and late peri-procedural DHF; namely, early peri-procedural DHF was defined as occurring within 2 days after the procedure and late peri-procedural DHF as occurring [?] 3 days after the procedure.

Long-term rhythm outcomes after catheter ablation were followed for 2 years. late phase DHF requiring re-hospitalization in this 2-year period was also assessed.

A 12-lead ECG and blood tests were performed one day before the procedure. In accordance with Japanese guidelines, we set cutoff values for brain natriuretic peptide (BNP) and N-terminal pro-BNP of $100 \mathrm{ng} / \mathrm{L}$ and $400 \mathrm{ng} / \mathrm{L}$, respectively. ${ }^{4}$ After the procedure, a 12-lead ECG was performed one day after the procedure, and $\Delta$ heart rate, which was defined as heart rate after ablation minus heart rate before ablation, were 
calculated. ECG monitoring, peripheral oxyhemoglobin saturation monitoring, and observations by nurses were performed for at least 2 days after the procedure. In general, ECGs and chest X-rays were also performed when DHF occurred. If heart rate could not be measured, pulse rate was measured and substituted for heart rate.

Followup after discharge was performed as a part of routine visits, usually conducted at $1,3,6,9,12,18$, and 24 months after the procedure. Each routine visit included a 12-lead ECG. In addition, 24-hour Holter electrocardiography was performed 6 months after the procedure.

Early recurrence of AF was defined as atrial tachyarrhythmias detected by 12-lead ECG or atrial tachyarrhythmias lasting $>30$ seconds detected by ECG monitoring or 24-hour Holter monitoring at $<90$ days after the procedure. If early recurrence of AF occurred, antiarrhythmic drugs were generally used. Electrical cardioversion was performed according to the chief doctor's judgment.

AF recurrence was defined as atrial tachyarrhythmias detected by 12-lead ECG or atrial tachyarrhythmias lasting $>30$ seconds detected by 24-hour Holter electrocardiography [?] 90 days after the procedure.

Statistical methods

Categorical data are presented as absolute values and percentages, and continuous data as mean \pm standard deviation or median (1st-3rd quartile). Tests for significance were conducted using the chi-squared test for categorical variables and the unpaired $t$-test or Mann-Whitney $U$-test for continuous variables. Univariate and multivariate Cox proportional hazards regression analyses were used to determine clinical factors associated with the DHF. Variables with a $P$ value [?] 0.05 in the univariate models were included in the multivariate analysis. Kaplan-Meier analysis, log-rank test, and Cox proportional hazards regression analyses were used to investigate the association between the AF recurrence or late phase DHF requiring re-hospitalization and DHF.

All analyses were performed using commercial software (SPSS version 25, SPSS, Inc., Chicago IL, USA).

\section{Results}

Patient and procedural characteristics

PV isolation was successfully completed in all 1004 cases, using Carto 3 in 822 (82\%), Ensite NavX in 159 (16\%) and Rhythmia in $23(2 \%)$. Patient characteristics are shown in Table 1. Procedural characteristics between patients with and without DHF are shown in Supplementary Table 1.

\section{$D H F$}

DHF occurred in 32/1004 (3\%) cases. Ten (31\%) cases developed early recurrence of AF before DHF. For management of early recurrence of $\mathrm{AF}$, antiarrhythmic agents were used in 8 cases and electrical cardioversion were performed in 3 cases. A representative case of DHF is shown in Figure 1. The time course and heart rate from the procedure to DHF are shown in Figure 2, and the details of patients with DHF are shown in Supplementary Table 2 .

There were different etiologies of heart failure. Patients with late peri-procedural DHF had a higher prevalence of valvular heart disease than those with early peri-procedural DHF (4 [40\%] versus $2[9 \%], P=0.04$ ). There was no difference in other presumed etiologies of heart failure, clinical scenarios, Nohria-Stevenson classification, or management of DHF between early peri-procedural DHF and late peri-procedural DHF.

Predictors of overall DHF are shown in Supplementary Table 3. Lower $\Delta$ heart rate after the procedure and early recurrence of AF were independent predictors of DHF on multivariate analysis.

Early peri-procedural DHF occurred in $22(2 \%)$ patients. Patients with early peri-procedural DHF had a higher prevalence of persistent AF, past history of symptomatic heart failure, diuretic usage, elevated BNP or N-terminal pro-BNP, and LVAs than those without early peri-procedural DHF. In addition, patients with early peri-procedural DHF had a higher New York Heart Association class, higher left atrial diameter, 
lower left ventricular ejection fraction, and lower $\Delta$ heart rate than those without early peri-procedural DHF. Lower $\Delta$ heart rate was also an independent predictor of early peri-procedural DHF in multivariate analysis (Table 2). Four (18\%) patients needed intensive care; however, no mechanical ventilation with endotracheal intubation for respiratory failure was needed. Two (9\%) patients needed temporary cardiac pacing for bradycardia. Duration of hospitalization after early peri-procedural DHF was 3 (1-9) days, and all patients were discharged from the hospital. Median New York Heart Association class at discharge was 1 (range, $1-1)$.

Late peri-procedural DHF occurred in 10 (1\%) of patients. Patients with late HF had a higher prevalence of early recurrence of AF, past history of symptomatic heart failure, diuretics usage, and LVAs than those without late peri-procedural DHF. In addition, patients with late peri-procedural DHF had a higher New York Heart Association class, higher $\mathrm{CHA}_{2} \mathrm{DS}_{2}$-VASc score, and lower left ventricular ejection fraction than those without late peri-procedural DHF. Early recurrence of AF was also an independent predictor of late peri-procedural DHF in multivariate analysis (Table 3). Two (20\%) patients needed intensive care; however, no mechanical ventilation with endotracheal intubation or temporary cardiac pacing was needed. Duration of hospitalization after late peri-procedural DHF was $8(2-27)$ days. One patient died due to progression of dilated cardiomyopathy; the other patients were discharged from the hospital. Median New York Heart Association class at discharge was 1 (range, 1-2).

$\Delta \eta \epsilon a \rho \tau \rho a \tau \epsilon a \nu \delta \Delta H \Phi$

$\Delta$ heart rate was an independent predictor of early peri-procedural DHF; therefore, subgroup analysis of $\Delta$ heart rate was also performed. Persistent AF, past history of symptomatic heart failure, low left ventricular ejection fraction, beta antagonist usage, and LVAs were significantly associated with lower $\Delta$ heart rate (Figure 3). In contrast, $\Delta$ heart rate was similar between patients with $\mathrm{AF}$ duration $<1$ year and those with AF duration [?] 1 year $(2 \pm 22$ versus $-1+-19$ beats/min, $P=0.17)$.

Impacts of peri-procedural DHF on long-term rhythm and cardiac outcomes.

During the 2-year study period, 277 (28\%) cases developed AF recurrence. Freedom from AF recurrence was similar between patients with and without DHF (hazard ratio 1.2, 95\% confidence interval 0.7-2.3, $\mathrm{p}=$ $0.50)$.

Seventeen $(2 \%)$ cases developed late phase DHF requiring re-hospitalization during the 2-year study period. Freedom from late phase DHF requiring re-hospitalization was significantly lower in patients with DHF than those without DHF (hazard ratio 11, 95\% confidence interval 3.6-34, p < 0.01) (Figure 4).

\section{Discussion}

In this retrospective study of 1004 patients undergoing initial AF ablation, we found that DHF which needed medical therapy during post-ablation hospitalization or re-hospitalization $<90$ days after the ablation occurred in $32(3 \%)$ patients. DHF frequently occurred in the first 48 hours after the procedure. Independent predictors of DHF were lower $\Delta$ heart rate and early recurrence of AF. In particular, lower $\Delta$ heart rate was an independent predictor of early peri-procedural DHF, which occurred within 2 days after the procedure, and early recurrence of AF was an independent predictor of late peri-procedural DHF, which occurred [?] 3 days after the procedure. Freedom from AF recurrence was similar between patients with and without DHF. In contrast, freedom from late phase DHF requiring re-hospitalization was significantly lower in patients with DHF than without DHF.

\section{Early peri-procedural DHF}

In this study, DHF occurred in $3 \%$ of patients. Previous studies showed a $3 \%$ incidence of pulmonary edema after electrical cardioversion, ${ }^{6}$ and showed that $20-26 \%$ of patients undergoing AF ablation suffered symptoms of heart failure within 30 days after the procedure. ${ }^{7,8}$

In our study, $\Delta$ heart rate was an independent predictor of early peri-procedural DHF. Either high heart rate before the procedure or decrease in heart rate after the procedure was associated with DHF. 
Although preprocedure tachycardia is a recognized independent prognostic risk factor for heart failure ${ }^{9}$ an increase in heart rate with increasing severity of heart failure is considered to be a compensatory response to the reduced cardiac reserve via activation of sympathetic activity. ${ }^{10,11}$ In addition, some AF patients develop tachycardia-induced cardiomyopathy. ${ }^{12}$ In tachycardia-induced cardiomyopathy patients, it takes $4-6$ weeks after sinus conversion to improve left ventricular ejection fraction. ${ }^{13}$ In these patients, low $\Delta$ heart rate might induce DHF.

Decrease in heart rate can occur after the procedure. In general, heart rate is higher in patients with AF than in those with sinus rhythm, ${ }^{14}$ and sinus node dysfunction frequently occurs in patients with AF. ${ }^{15}$ Sedation during the procedure also results in a decrease in heart rate. ${ }^{16}$ These factors lead to DHF immediately after ablation, that is, early peri-procedural DHF.

\section{Late peri-procedural DHF}

Our study showed that $9(90 \%)$ patients developed early recurrence of AF before late peri-procedural DHF. AF results in a rapid ventricular heart rate. ${ }^{17}$ In addition, loss of effective atrial contractile function occurs. ${ }^{17}$ These factors lead to a reduction in cardiac output, so AF is a risk factor for DHF. ${ }^{17}$ Our study also showed early recurrence of AF was an independent predictor of DHF, especially of late peri-procedural DHF. A previous study showed that $78 \%$ of early recurrence of AF occurred within 7 days after catheter ablation. ${ }^{18}$ It is thought that early recurrence of AF often occurs at some point and contributes to late peri-procedural DHF.

\section{DHF and late phase DHF requiring re-hospitalization}

During the 2-year study period, freedom from late phase DHF requiring re-hospitalization was significantly lower in patients with a DHF than in those without. Past history of hospitalization due to DHF predicts cardiovascular death and re-hospitalization due to DHF. Once symptomatic heart failure occurs, the stage of heart failure and underlying structural heart diseases has already progressed. ${ }^{4}$ Diuretics and inotropic agents often used for management of DHF can cause renal impairment and myocardial toxicity, and might lead to late phase DHF requiring re-hospitalization in the long term. ${ }^{19,20}$ Careful treatment, lifestyle management, and close followup of chronic heart failure will be needed in patients with DHF after ablation.

\section{Clinical implications}

Observation of $\Delta$ heart rate and early recurrence of AF are simple and noninvasive, and prediction of DHF after catheter ablation may be useful for safe peri-procedural management. If $\Delta$ heart rate is low, close observation of symptoms is needed in the early phase (within 2 days) after the procedure. Additionally, quick management of early recurrence of $\mathrm{AF}$, such as antiarrhythmic agents and electrical cardioversion, is taken into consideration in the late phase ([?] 3 days) after the procedure.

\section{Limitations}

Several limitations of our study warrant mention. First, although ECG monitoring was performed for at least two days after the procedure, we could not observe heart rate after discharge. Second, we were unable to assess for mild DHF that did not require re-hospitalization, and the indication for re-hospitalization due to DHF depended on the chief doctor's judgment. Third, the infusion volume in the peri-procedural period and ablation procedure might have varied, even though we attempted to standardize as far as possible. Fourth, we could not fully eliminate confounding factors because this study was retrospective, and there were therefore some differences in patient characteristics between patients with DHF and those without. Finally, the number of cases of DHF was small, weakening the statistical analysis.

\section{Conclusions}

In patients with $\mathrm{AF}$, DHF occurred in $3 \%$ of patients after catheter ablation. Heart rate decrease after the procedure and early recurrence of AF were independent predictors of DHF after catheter ablation. In particular, heart rate decrease after the procedure was an independent predictor of early peri-procedural $\mathrm{DHF}$ and early recurrence of AF was an independent predictor of late peri-procedural DHF. 


\section{Acknowledgment}

None.

\section{References}

1. Calkins H, Hindricks G, Cappato R, Kim YH, Saad EB, Aguinaga L, Akar JG, Badhwar V, Brugada J, Camm J, Chen PS, Chen SA, Chung MK, Cosedis Nielsen J, Curtis AB, Davies DW, Day JD, d'Avila A, Natasja de Groot NMS, Di Biase L, Duytschaever M, Edgerton JR, Ellenbogen KA, Ellinor PT, Ernst S, Fenelon G, Gerstenfeld EP, Haines DE, Haissaguerre M, Helm RH, Hylek E, Jackman WM, Jalife J, Kalman JM, Kautzner J, Kottkamp H, Kuck KH, Kumagai K, Lee R, Lewalter T, Lindsay BD, Macle L, Mansour M, Marchlinski FE, Michaud GF, Nakagawa H, Natale A, Nattel S, Okumura K, Packer D, Pokushalov E, Reynolds MR, Sanders P, Scanavacca M, Schilling R, Tondo C, Tsao HM, Verma A, Wilber DJ, Yamane T; Document Reviewers:. 2017 HRS/EHRA/ECAS/APHRS/SOLAECE expert consensus statement on catheter and surgical ablation of atrial fibrillation. Europace. 2018 ; 20:e1-e160.

2. Deshmukh A, Patel NJ, Pant S, Shah N, Chothani A, Mehta K, Grover P, Singh V, Vallurupalli S, Savani GT, Badheka A, Tuliani T, Dabhadkar K, Dibu G, Reddy YM, Sewani A, Kowalski M, Mitrani R, Paydak H, Viles-Gonzalez JF. In-hospital complications associated with catheter ablation of atrial fibrillation in the United States between 2000 and 2010: analysis of 93801 procedures. Circulation. 2013; 128:2104-12.

3. Jones DG, Haldar SK, Hussain W, Sharma R, Francis DP, Rahman-Haley SL, McDonagh TA, Underwood SR, Markides V, Wong T. A randomized trial to assess catheter ablation versus rate control in the management of persistent atrial fibrillation in heart failure. J Am Coll Cardiol. 2013; 61: 1894-903.

4. Tsutsui H, Isobe M, Ito H, Ito H, Okumura K, Ono M, Kitakaze M, Kinugawa K, Kihara Y, Goto Y, Komuro I, Saiki Y, Saito Y, Sakata Y, Sato N, Sawa Y, Shiose A, Shimizu W, Shimokawa H, Seino Y, Node K, Higo T, Hirayama A, Makaya M, Masuyama T, Murohara T, Momomura SI, Yano M, Yamazaki K, Yamamoto K, Yoshikawa T, Yoshimura M, Akiyama M, Anzai T, Ishihara S, Inomata T, Imamura T, Iwasaki YK, Ohtani T, Onishi K, Kasai T, Kato M, Kawai M, Kinugasa Y, Kinugawa S, Kuratani T, Kobayashi S, Sakata Y, Tanaka A, Toda K, Noda T, Nochioka K, Hatano M, Hidaka T, Fujino T, Makita S, Yamaguchi O, Ikeda U, Kimura T, Kohsaka S, Kosuge M, Yamagishi M, Yamashina A; Japanese Circulation Society and the Japanese Heart Failure Society Joint Working Group. for the Japanese Circulation Society and the Japanese Heart Failure Society Joint Working Group. JCS 2017/JHFS 2017 Guideline on Diagnosis and Treatment of Acute and Chronic Heart Failure-Digest Version. Circ J. 2019; 83: 2084-2184.

5. Upshaw CB Jr. Hemodynamic changes after cardioversion of chronic atrial fibrillation. Arch Intern Med. $1997 ; 157: 1070-6$.

6. Resnekov L, McDonald L. Complications in 220 patients with cardiac dysrhythmias treated by phased direct current shock, and indications for electroconversion. Br Heart J. 1967; 29: 926-36.

7. Huang HD, Waks JW, Contreras-Valdes FM, Haffajee C, Buxton AE, Josephson ME. Incidence and risk factors for symptomatic heart failure after catheter ablation of atrial fibrillation and atrial flutter. Europace 2016; 18:521-30.

8. Gilge JL, Ahmed A, Clark BA, Slaten A, Devathu R, Olson JA, Padanilam BJ, Nair GV, Joshi SA, Ravichandran AK, Patel PJ. Left atrial hypertension and the risk of early incident heart failure after atrial fibrillation ablation. J Cardiovasc Electrophysiol. 2021; 32: 325-32.

9. Nanchen D, Leening MJ, Locatelli I, Cornuz J, Kors JA, Heeringa J, Deckers JW, Hofman A, Franco $\mathrm{OH}$, Stricker BH, Witteman JC, Dehghan A. Resting heart rate and the risk of heart failure in healthy adults: the Rotterdam Study. Circ Heart Fail 2013; 6: 403-10.

10. Okada S, Iwahana T, Kobayashi Y. Is High Heart Rate Always Harmful to Heart Failure Patients? Circ J 2020; 84: 1673-74.

11. Tsutsui H, Ide T, Kawasaki Y. Is High Heart Rate Always Harmful to Heart Failure Patients? - Reply. Circ J 2020; 84: 1674-75.

12. January CT, Wann LS, Alpert JS, Calkins H, Cigarroa JE, Cleveland JC Jr, Conti JB, Ellinor PT, 
Ezekowitz MD, Field ME, Murray KT, Sacco RL, Stevenson WG, Tchou PJ, Tracy CM, Yancy CW; American College of Cardiology/American Heart Association Task Force on Practice Guidelines. 2014 AHA/ACC/HRS guideline for the management of patients with atrial fibrillation: a report of the American College of Cardiology/American Heart Association Task Force on Practice Guidelines and the Heart Rhythm Society. J Am Coll Cardiol 2014; 64: e1-76.

13. Zimmermann AJ, Bossard M, Aeschbacher S, Schoen T, Voellmin G, Suter Y, Lehmann A, Hochgruber T, Pumpol K, Sticherling C, Kühne M, Conen D, Kaufmann BA. Effects of sinus rhythm maintenance on left heart function after electrical cardioversion of atrial fibrillation: implications for tachycardiainduced cardiomyopathy. Can J Cardiol 2015; 31: 36-43.

14. Keteyian SJ, Ehrman JK, Fuller B, Pack QR. Exercise Testing and Exercise Rehabilitation for Patients With Atrial Fibrillation. J Cardiopulm Rehabil Prev 2019; 39: 65-72.

15. Hocini M, Sanders P, Deisenhofer I, Jaïs P, Hsu LF, Scavée C, Weerasoriya R, Raybaud F, Macle L, Shah DC, Garrigue S, Le Metayer P, Clémenty J, Haïssaguerre M. Reverse remodeling of sinus node function after catheter ablation of atrial fibrillation in patients with prolonged sinus pauses. Circulation. 2003;108: $1172-5$.

16. Sairaku A, Yoshida Y, Hirayama H, Nakano Y, Ando M, Kihara Y. Procedural sedation with dexmedetomidine during ablation of atrial fibrillation: a randomized controlled trial. Europace. 2014 ;16:994-999.

17. Anter E, Jessup M, Callans DJ. Atrial fibrillation and heart failure: treatment considerations for a dual epidemic. Circulation. 2009;119:2516-25.

18. Oka T, Koyama Y, Tanaka K, Hirao Y, Tanaka N, Okada M, Yoshimoto I, Kitagaki R, Okamura A, Iwakura K, Sakata Y, Fujii K, Inoue K. Early recurrence during the blanking period and left atrial reverse remodeling after catheter ablation for non-paroxysmal atrial fibrillation. Int J Cardiol Heart Vasc. 2020 Jul 23;30:100588.

19. Gheorghiade M, Zannad F, Sopko G, Klein L, Piña IL, Konstam MA, Massie BM, Roland E, Targum S, Collins SP, Filippatos G, Tavazzi L; International Working Group on Acute Heart Failure Syndromes. Acute heart failure syndromes: current state and framework for future research. Circulation. 2005;112:3958-68.

20. Silver MA, Horton DP, Ghali JK, Elkayam U. Effect of nesiritide versus dobutamine on short-term outcomes in the treatment of patients with acutely decompensated heart failure. J Am Coll Cardiol. 2002; 39:798-803.

Figure legendsFigure 1. Representative case of DHF after AF ablation In this patient, DHF occurred 12 hours after RFCA for persistent AF. Intensive care, diuretics, and nitrates were needed for management. AF: atrial fibrillation, RFCA: radiofrequency catheter ablation, DHF: decompensated heart failureFigure 2. Time course and heart rate from the procedure to DHF DHF occurred in $32(3 \%)$ of all patients, and $10(31 \%)$ patients developed early recurrence of AF before DHF. AF: atrial fibrillation, DHF: decompensated heart failureFigure 3. Subgroup analysis of $\Delta$ heart rate Persistent AF, past history of symptomatic heart failure, low LVEF, beta-blocker usage and low-voltage areas were significantly associated with lower $\Delta$ heart rate AF: atrial fibrillation, LVEF: left ventricular ejection fractionFigure 4. Freedom from AF recurrence and late phase DHF requiring re-hospitalization in patients with and without DHF Freedom from AF recurrence was similar between patients with and without DHF. In contrast, freedom from late phase DHF requiring re-hospitalization was significantly lower in patients with DHF than in those without. AF: atrial fibrillation, DHF: decompensated heart failure

\section{Hosted file}

Matsuda DHF_ablation_table_JCE.docx available at https://authorea.com/users/311170/articles/ 527022-clinical-risk-factors-for-decompensated-heart-failure-after-atrial-fibrillationablation 
$\checkmark 69$ year-old male

$\checkmark$ Height: $170 \mathrm{~cm}$, Body weight: $107 \mathrm{~kg}$, Body mass index: $37 \mathrm{~kg} / \mathrm{m}^{2}$

$\checkmark$ Past history of symptomatic heart failure (-), Left ventricular ejection fraction: $49 \%$

$\checkmark$ Comorbidity: Chronic kidney disease, Diabetes, Dyslipidemia

$\checkmark$ AF duration: Persistent AF lasting for 1 month
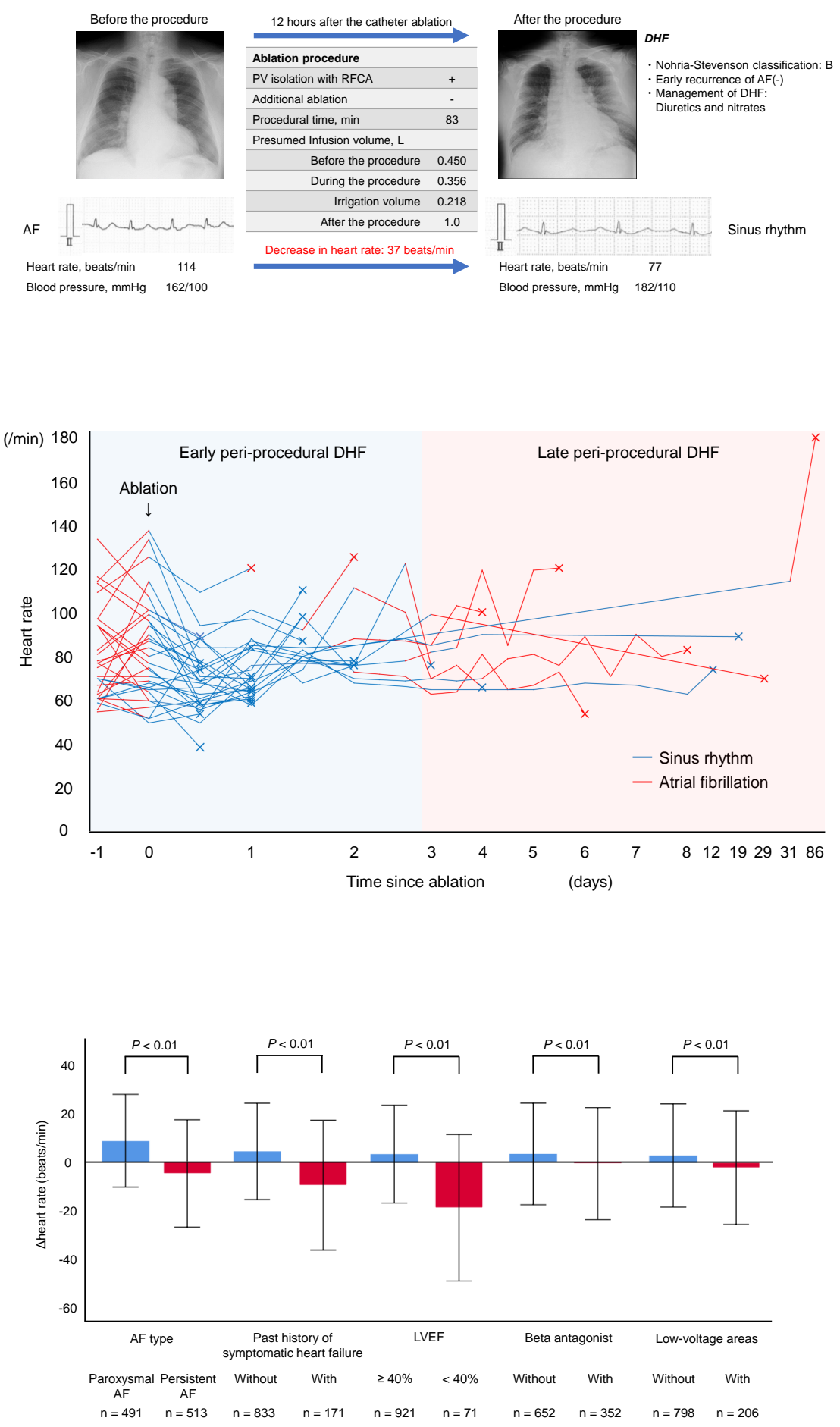
(A) Freedom from AF recurrence

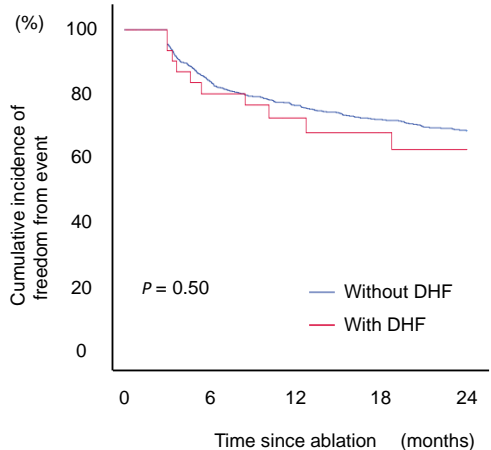

No. at risk

$\begin{array}{llllll}\text { With DHF } & 32 & 23 & 16 & 14 & 8\end{array}$

(B) Late phase DHF requiring re-hospitalization

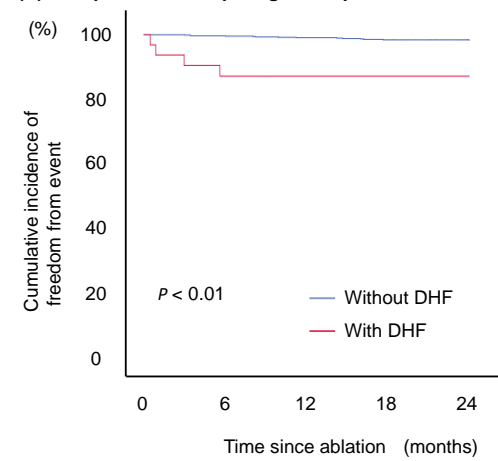

No. at risk

$\begin{array}{ccccc}32 & 25 & 20 & 17 & 9 \\ 972 & 912 & 814 & 651 & 470\end{array}$

